\title{
$\mathrm{C}$ OMMENTARY
}

\section{Outpatient thromboprophylaxis after hip or knee surgery: discrepancies and concerns}

\author{
William D. Fisher MD, Alexander G.G. Turpie MD
}

$\infty$

See related article page $\mathrm{I} 545$

$\mathrm{T}$ he high risk of venous thromboembolism among patients undergoing major lower limb surgery is well recognized. Recommendations for appropriate prophylaxis based on solid scientific evidence have been published by the American College of Chest Physicians on a regular basis since $1986 .{ }^{1}$ Similar guidelines have been developed by other expert groups. ${ }^{2}$ Because of the perceived risk of increased postoperative bleeding, there have been concerns among the orthopedic surgical community about the use of prophylactic anticoagulant therapy. Although comparisons with untreated patients are few, the evidence suggests bleeding is not increased. ${ }^{3}$ Nevertheless, these concerns led the American Academy of Orthopaedic Surgeons to develop alternative guidelines,${ }^{4}$ which are based on data supporting the reduction of clinical outcomes, namely pulmonary emboli, rather than all venous thromboembolism events.

The guidelines from the American College of Chest Physicians recommend a minimum of Io days of anticoagulant prophylaxis. ${ }^{1}$ In the past, anticoagulant prophylaxis was often given to the patient during his or her hospital stay but was discontinued on discharge. Ultrasonographic screening has been used to support the discontinuation of prophylaxis; however, this approach has poor predictive value and is no longer recommended for use in this situation. ${ }^{5}$ With the increasingly short hospital stays after joint-replacement surgery, it is no longer appropriate to consider prophylaxis for in-hospital use alone, and patients are frequently advised to continue prophylaxis after discharge. The American College of Chest Physicians guidelines strongly recommend extending prophylaxis to up to 35 days for total hip replacement and hip fracture. This length of prophylaxis is not recommended after total knee replacement because the peak incidence of deep vein thrombosis is earlier and the risk-benefit ratio is correspondingly lower.

In this issue of CMAJ, Rahme and colleagues report variation in the use of postdischarge thromboprophylaxis after hip- or knee-replacement surgery among patients aged 65 years and older after discharge. ${ }^{6}$ Over $80 \%$ of these patients did not receive thromboprophylaxis after discharge. There appears to be increased risk of short-term mortality among

\section{Key points}

- There is unexplained variation in compliance with accepted guidelines for thromboembolism prophylaxis after orthopedic surgery.

- Failure to provide recommended prophylaxis appears to be associated with short-term increases in mortality.

- There is a need for better strategies such as standardized care plans and preprinted orders to ensure that appropriate care is continued after hospital discharge.

patients who did not receive postdischarge thromboprophylaxis compared with those who did.

This is an important observation that further emphasizes the need for greater awareness of the importance of evidence-based prophylaxis in high-risk surgical populations. This was also highlighted by the results of the ENDORSE study, which was a multinational study that reported a wide variation in appropriate use of prophylactic therapy among countries and specialties.

However, the study by Rahme and colleagues has a number of limitations that reduce its impact. Their study included only $33 \%$ of all of the patients who underwent hip and knee surgery who were discharged directly from hospital, and it excluded prescriptions given to patients while in hospital or rehabilitation centre. Thus, their findings may not be generalizable. Furthermore, their study included 3 markedly different groups of patients: those who received hip-replacement surgery, knee-replacement surgery or hip-fracture surgery. The timing of occurrence of venous thromboembolism events varies among these groups of patients. The peak incidence of symptomatic deep venous thrombosis is at $\mathrm{I} 6$ days after a total knee replacement but is at 27 days after a hip replacement and 35 days after a hip fracture repair. ${ }^{8}$ The expected mortality among these groups also differs markedly. Although the mortality at 3 months for elective hip- and kneereplacement arthroplasty may be as low as the reported

From McGill University Health Centre, Montréal, Que. (Fisher), and McMaster University, Hamilton, Ont (Turpie). 
$0.3 \%-0.6 \%$ in the study by Rahme and colleagues, ${ }^{6}$ the 3 -month mortality after hip-fracture surgery can be as high as $10 \% .{ }^{9}$ This high figure is almost certainly the result of the associated comorbidities among those in the hip-fracture group.

In Canada, $70 \%$ of orthopedic surgeons from each province (but only $44 \%$ of orthopedic surgeons from Quebec) contribute to the central Canadian Joint Replacement Registry. The 2007 report from this registry suggests that compliance with approved forms of prophylaxis is as high as $97 \% .{ }^{10}$ In total, $70 \%$ of surgeons prescribed low-molecular-weight heparin; however, its length of use or postdischarge use was not reported, nor were provincial variations recorded. The data presented by Rahme and colleagues suggest that (at least within the Quebec orthopedic community) there was not full compliance with the recommended standards. It appears that, almost independent of the length of hospital stay, the percentage of patients who continued thromboprophylaxis after discharge was about $20 \%$. Unfortunately, the number of patients who received some form of inpatient prophylaxis was not reported because individual hospital data are not available in the Quebec health care system. Even if we assume that $100 \%$ compliance was achieved in hospital, the number of patients who continue prophylaxis after discharge is clearly not ideal.

The inclusion of patients with hip fractures in the study by Rahme and colleagues adds a confounding factor to the results. Although these patients are at equal risk of venous thromboembolism, they also have other medical comorbidities, and mortality in this group is significantly higher than among those undergoing elective joint replacement surgery. This is clearly confirmed within this study, but it does mean that there are much smaller numbers on which to base conclusions about short-term mortality. The number of deaths after total hip replacement was 27 and was even lower (II patients) after total knee replacement. The mortality rates $(0.6 \%$ and $0.3 \%$ respectively) are, however, comparable with other studies. ${ }^{11}$

It is difficult to draw many conclusions from the data presented by Rahme and colleagues about the risks from inadequate use of prophylaxis. There were too few deaths to postulate extensively about the risk of short-term mortality without additional information about in-hospital care and causes of death. This study's strength, however, lies in the completeness of the data about this select group of patients.

Failure to continue prophylaxis after discharge may be the result of several factors. The physician may feel that the patient's increased mobility after discharge home reduces the risk of venous thromboembolism. This risk, however, exists for up to 3 months after surgery, with the majority of events occurring after discharge. ${ }^{11}$ Patient compliance with selfinjection or difficulty in arranging home care may be at fault.
Finally, for some patients, the cost of outpatient prescriptions may be a factor in the decision to continue prophylaxis.

A critical factor in eliminating noncompliance may be the establishment of standard hospital prophylaxis policies and the introduction of preprinted medication orders. A similar policy, which includes the use of low-molecular-weight heparin after vascular surgery, has been successful, and is based on a team approach that includes nursing, surgical and pharmacist participation..$^{12}$ Of equal importance is patient education and the inclusion of the standard protocol in the informed consent.

Competing interests: William Fisher has served as a consultant for and has received travel assistance from Bayer and Sanofi-Aventis. Alexander Turpie has served as a consultant for and has received speaker's fees from Sanofi-Aventis, Bayer and GlaxoSimthKline.

Contributors: Both of the authors contributed to the conception and design of the article, revised it critically and approved the final version for publication.

\section{REFERENCES}

I. Geerts WH. Pineo GF. Heit JA, et al. Prevention of venous thromboembolism: the Seventh ACCP Conference on Antithrombotic and Thrombolytic Therapy. Chest 2004;126;312-34.

2. Cardiovascular Disease Educational and Research Trust; Cyprus Cardiovascular Disease Educational and Research Trust; European Venous Forum; et al. Prevention and treatment of venous thromboembolism. International consensus statement (guidelines according to scientific evidence). Int Angiol 2006;25:10I-6I.

3. American Academy of Orthopaedic Surgeons clinical guidelines on prevention of symptomatic pulmonary embolism in patients undergoing total hip or knee arthroplasty. Available: http://www.aaos.org/Research/guidelines/PE_guideline .pdf (accessed 2008 Apr 25).

4. Leclerc JR, Geerts WH, Desjardins L, et al. Prevention of deep vein thrombosis after major knee surgery — a randomized double-blind trial comparing a low molecular heparin fragment (enoxaparin) to placebo. Thromb Haemost 1992;67:417-23.

5. Robinson KS, Anderson DR, Gross M, et al. Ultrasonographic screening before hospital discharge for deep venous thrombosis after arthroplasty: the post-arthroplasty screening study. A randomized controlled trial. Ann Intern Med I997;127:439-45.

6. Rahme E, Dasgupta K, Burman M, et al. Postdischarge thromboprophylaxis and mortality risk after hip- or knee-replacement surgery. CMAJ 2008;178:1545-54.

7. Cohen AT, Tapson VF, Bergmann J-F, et al. Venous thromboembolism risk and prophylaxis in the acute hospital care setting (ENDORSE study): a multinational cross-sectional study. Lancet 2008;371:387-94.

8. Dahl OE, Gudmundsen TE., Haukeland L. Late occurring clinical deep vein thrombosis in joint-operated patients. Acta Orthop Scand 2000;71:47-50.

9. White BL, Fisher WD, Laurin CA. Rate of mortality for elderly patients after fracture of the hip in the I980's. J Bone Joint Surg Am I987;69:1335-40.

Io. The Canadian Joint Replacement Registry 2007 Annual Report on Hip and Knee Replacements in Canada. Available: http://secure.cihi.ca/cihiweb/dispPage.jsp ?cw_page=PG_835_E\&cw_topic=835\&cw_rel=AR_30_E (accessed 2008 May 2).

II. White RH, Romano PS, Zhou H, et al. Incidence and time course of thromboemblolic outcomes following total hip or knee arthroplasty. Arch Intern Med 1998; I58:1525-3I.

I2. Gramse CA, Hingorani A, Ascher E. Postoperative anticoagulation in vascular surgery-part two: A summery of lessons learned in our successful discharge planning experience using enoxaparin after vascular surgery. J Vasc Nurs 2003;2I:I24-3I.

Correspondence to: Dr. William D. Fisher, McGill University Health Centre, I650 Cedar Ave., Bureau B5.158.7, Montréal QC $\mathrm{H}_{3} \mathrm{G}_{\mathrm{IA}}$; fax 514 934-8283; wfisher@sympatico.ca 